\title{
APPLICATION OF MILLISECOND PULSED LASER WELDING IN MEMS PACKAGING
}

\author{
P. Bozorgi", C.B. Burgner, Z. Yie, C. Ding, K.L. Turner and N.C. MacDonald \\ Department of Mechanical Engineering, University of California Santa Barbara, California, USA
}

\begin{abstract}
This paper reports a new packaging method for a wide range of MEMS applications on both the wafer and device scale. Titanium is used as the packaging material in this work and both Si-MEMS and Ti-MEMS devices are integrated into a $350 \mu \mathrm{m}$ titanium substrate. A Nd:YAG pulsed laser is used as a localized heating source to micro-weld a $350 \mu \mathrm{m}$ titanium cap to the substrate. Simulation of the heat conduction of a two-dimensional time-dependent pulse laser between the substrate and cap was carried out using COMSOL to investigate pulsed laser melting properties. To avoid thermal distortion of the welding, several geometries at the cap and substrate interface were investigated to minimize laser intensity in order to achieve the required melting depth.
\end{abstract}

\section{INTRODUCTION}

Packaging provides structural and environmental protection for MEMS devices to enhance their reliability but poses a critical challenge for the commercialization of MEMS products. Techniques that are compatible with wafer level fabrication, low temperature processing, vacuum and hermetic encapsulation, and standard MEMS post-fabrication approaches are needed in many applications.

Although $\mathrm{Au}-\mathrm{Au}$ and $\mathrm{Si}-\mathrm{Au}$ eutectic bonding [1] and anodic bonding [2] have been widely used in MEMS packaging, these global heating packaging approaches still have several drawbacks. They are not reproducible, have surface and intermediate film dependency, and require various high temperature steps for bonding. As such, no temperature sensitive material survives through the bonding process.

In this work, we use titanium as the packaging material with a pulsed laser to locally heat and weld a titanium cap to the substrate. The proposed method addresses the drawbacks of eutectic and anodic bonding approaches. Titanium has been studied as a new material to produce MEMS [3]. This paper introduces titanium as an advantageous MEMS packaging material due to its high fracture toughness, weldability and potential in vivo applications in biotechnology [4].

\section{APPROACH AND MODELING}

When a laser beam is irradiated onto the surface of a material, the absorbed energy causes heating, melting and evaporation of the material depending on the absorbed laser power intensity [5]. Laser welding is performed by moving the focused beam over the surface of the work piece along the desired contour that separates the two pieces to be joined. If the laser beam intensity is sufficiently high and the scanning speed is not too large, evaporation throughout the full depth of the workpiece can be obtained and the so called keyhole is formed, see Figure 1. This keyhole absorbs a considerable amount of the laser beam power. Thus, the keyhole plays an important role in transferring and distributing the laser energy deep into the material and provokes melt depth, Figure 1 [6]. The thickness $h$ of a laser-induced melting layer is an important parameter in pulsed laser interaction with the material surface. It is a key factor for quantifying the hydrodynamic aspect of laser ablation and hermiticity of laser welding.

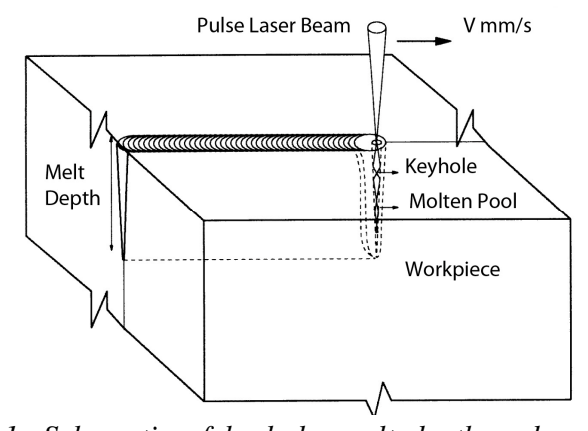

Figure 1: Schematic of keyhole, melt depth and molten pool in pulse laser welding.

Previous studies on the melt depth of pulsed laser [7] showed that the thickness $h$ is a function of many parameters

$$
h \propto\left(I, \tau, \alpha, A, \chi, C, \varphi, q, T_{m}, T_{b,}, T_{i}, D\right),
$$

where $I$ is the laser intensity, $\tau$ is the pulse duration, $\alpha$ is the absorption coefficient, $A$ is the surface absorptivity, $\chi$ is the thermal diffusivity, $C$ is the specific heat, $\varphi$ is the angle of incidence, $q$ is the latent heat of melting, $T_{m}$ and $T_{b}$ are the melting and boiling points, $T_{i}$ is the initial temperature, and $D$ is the laser focal spot size.

Hermiticity of the proposed packaging method is directly related to the melt depth of the titanium cap to the substrate in welding. The melted titanium at the interface of the cap and substrate is merged and solidified afterwards. Increasing the melt depth will generate a bigger molten pool at the interface and consequently stronger bonding between the cap and substrate. We assumed in our simulation the material properties during the welding stay constant, therefore the laser intensity plays a main role in increasing the melt depth. However, increasing the laser intensity generates more heat during the welding and this raises heat distortion issues.

Optimization of melt depth requires knowledge of the behavior of $h$ over a wide range of the parameters. As a first step, vaporization effects are not considered in our simulation. We present a 2D time and spatial heat conduction model, Equation (2), in COMSOL to determine the melt depth and generated heat for various laser parameters such as intensity, pulse duration, pulse frequency, laser focal spot size, and welding speed. The correlation between the melt depth and generated heat was investigated to optimize $h$ based on minimizing the heat

$$
\frac{\partial}{\partial x}\left(k_{x} \frac{\partial T}{\partial x}\right)+\frac{\partial}{\partial y}\left(k_{y} \frac{\partial T}{\partial y}\right)+I=\rho c\left(\frac{\partial T}{\partial t}-v \frac{\partial T}{\partial x}\right),
$$

where $k_{x}$ and $k_{y}$ are thermal conductivity in $x$ and $y$ directions, $I$ is laser power per unit volume, $\rho$ is density, $t$ is time and $V$ is velocity of the laser welder.

As shown in Figure 2, the Nd:YAG pulse laser was modeled as a Gaussian heat source. The laser has a rectangle pulse shape and the heat source is turned on and off with frequency of pulse laser. The focal size of the laser was determined in accordance 
with the cap thickness as prescribed in [8]. The titanium cap and substrate are $350 \mu \mathrm{m}$ thick and the laser focal spot size is $300 \mu \mathrm{m}$, and the pulse duration is $1 \mathrm{~ms}$ with a frequency of $14 \mathrm{~Hz}$. Initial simulation results show a linear relationship between the laser intensity and temperature along the cross-section of the cap and substrate, Figure 2. Simulation result shows a pulse energy of $6 \mathrm{~J}$ is required to melt the substrate at the interface. The pulse generates $6 \mathrm{~kW}$ of heat which can be harmful to any MEMS device.
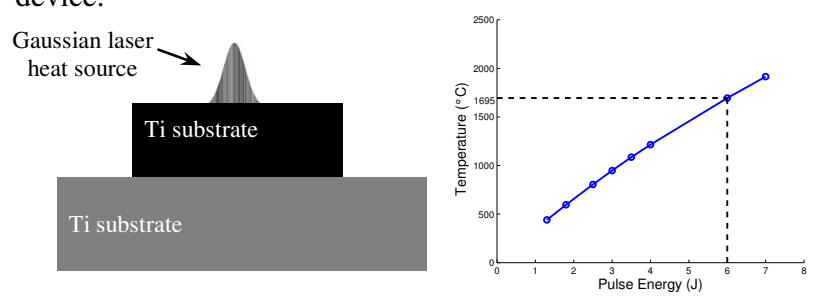

Figure 2: The required pulse energy to weld $300 \mu \mathrm{m}$ Ti cap to the substrate was determined by modeling laser as a Gaussian heat source.

Simulations seen in Figure 3 show the thickness and geometry of the cap and substrate at the interface play a significant role in determining laser parameters. In order to balance the melt depth and generated heat, three different bridge geometries were investigated for the substrate at the interface and the cap thickness was decreased to $100 \mu \mathrm{m}$. The simulation results in Figure 3(c) reveal that decreasing the bridge width and adding a trench reduces the power required for welding, thus reducing the unwanted heat transferred to the enclosed MEMS device. From these results, it is expected we achieve deeper melt depth on the narrow bridge with a trench for a given pulse laser compared to bridge configurations without a trench. Specifically, Figure 4(c) shows that changing the trench depth, $d$, from 20 to $10 \mu \mathrm{m}$ does not impact the temperature of the substrate at the interface. But changing the trench width from 100 to $250 \mu \mathrm{m}$ causes an increase in the temperature of the substrate from 3860 to $4600{ }^{\circ} \mathrm{C}$.

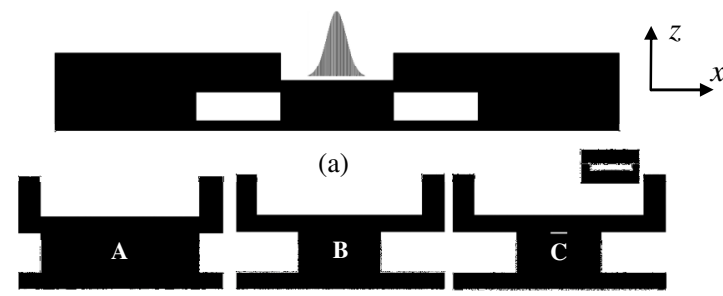

(b)

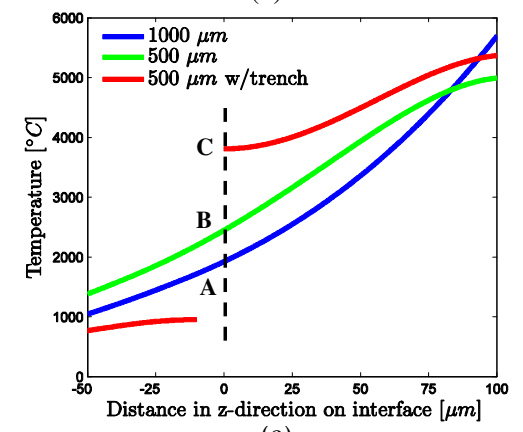

(c)

Figure 3: (a) Applied pulse laser with energy o $1.3 \mathrm{~J}$ and $1 \mathrm{~ms}$ pulse duration,(b) bridge $A$ and $B$ have a widths of 1000, $500 \mu \mathrm{m}$ respectively and bridge $C$ has width of $500 \mu \mathrm{m}$ with a trench (c) temperature gradient on the cap and the interface for each bridges.

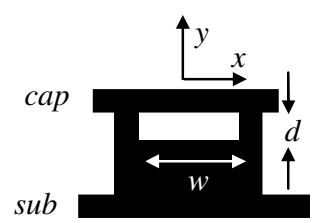

(a)

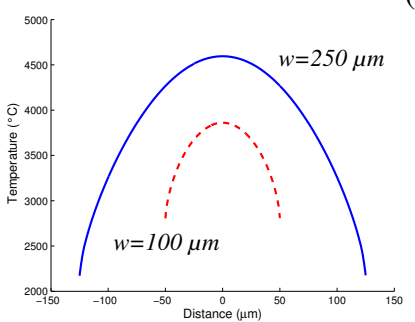

(b)

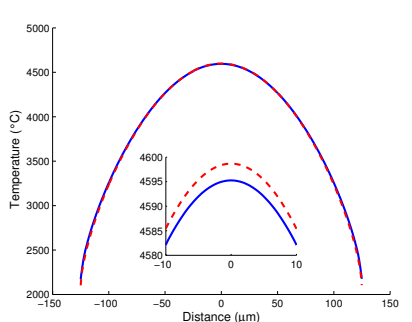

(c)
Figure 4: (a) The trench dimension on the substrate (b) Temperature gradient on the cap at the interface when the trench depth is $20 \mu \mathrm{m}$ and width is changed from 100 to $250 \mu \mathrm{m}$. (c) temperature gradient on the substrate does not change notably when the depth is increase from 10 to $20 \mu \mathrm{m}(d=250 \mu \mathrm{m})$.

As shown in Figure 5, welding the cap to the substrate causes a bending down of the cap at the interface to meet the substrate. This residual bending stress can generate micro cracks at the interface. To avoid this problem, the trench width should be increased from 100 to $250 \mu \mathrm{m}$. By increasing the width of the trench, a deeper melt depth occurs, Figure 4(b), and prevents cracks at the interface.

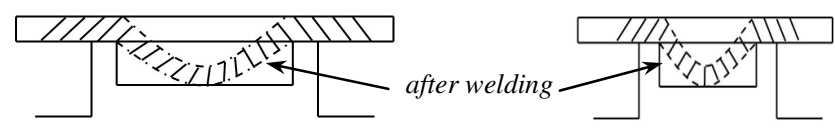

(a)

(b)

Figure 5: Schematic of the cap curvature after bending in welding for a trench with (a) $250 \mu \mathrm{m}$ width (b) $100 \mu \mathrm{m}$ width.

Simulation results in Figure 6 reveal that the temperature gradient in the substrate determines what distance from the heat source the MEMS device can be mounted. It can be seen that at a distance of $400 \mu \mathrm{m}$ from the laser heat source, the temperature on the substrate drops to $100{ }^{\circ} \mathrm{C}$ and the MEMS device can be safely mounted beyond this point.

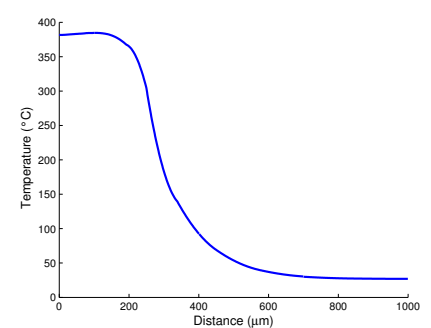

Figure 6: Temperature of substrate in welding process drops to $100{ }^{\circ} \mathrm{C}$ at a distance of $400 \mu \mathrm{m}$ from the laser spot. 


\section{FABRICATION AND EXPERIMENT}

In order to verify the simulation results on a bridge with a trench and show that creating a trench increases the melt depth, several experiments were carried out. The designed bridges on the substrate were fabricated via macro-machining and various laser parameters were applied to weld the cap to the substrate. Both the cap and substrate were taken from $350 \mu \mathrm{m}$ thick titanium wafers. Figure 7 presents a schematic of the fabrication process. The cap was macro-machined down to a thickness of $100 \mu \mathrm{m}$, Figure 7(a). Figure 7(b) shows the macro machining fabrication process of three bridges. The first two bridges have a width of 1000 and 500 $\mu \mathrm{m}$, respectively. The third bridge has a width of $500 \mu \mathrm{m}$ with a trench that is $250 \mu \mathrm{m}$ wide and $20 \mu \mathrm{m}$ deep. The cap and substrate were mated firmly, as presented in Figure 7(c). A millisecond YAG pulsed laser with a focal spot size of $300 \mu \mathrm{m}$, a pulse energy of $0.8 \mathrm{~J}$ and a pulse duration of $1 \mathrm{~ms}$ was used to weld the titanium cap to the substrate.

(a)
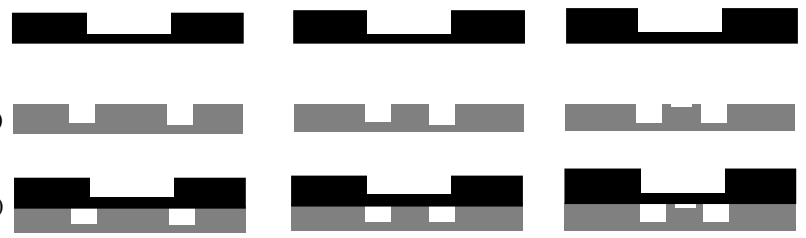

Figure 7: (a) Macro-machining of the titanium cap (b) Macromachining of the titanium substrate to fabricate the bridges (c)brining the cap and substrate together using vacuum chuck.

The experimental results in Figure 8 show that for the bridges without a trench, the cap and substrate were not solidified at their interface and no melt residue was seen on the substrate. But as shown in Figure 10(d) in the bridge with a trench, the cap and substrate were welded at their interface and melt residue was clearly seen on the substrate.
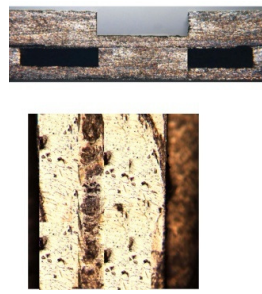

(b)

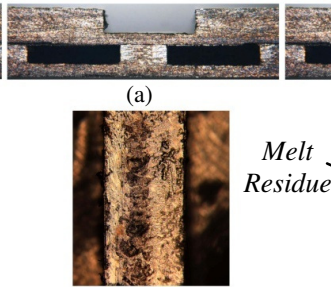

(c)
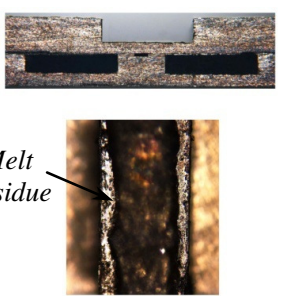

(d)
Figure 8: (a) Fabricated bridges before welding (b) and (c) The bridge with width of 1000 and $500 \mu \mathrm{m}$, respectively. A burnt line of welding can be seen on substrate (d) Showing the bridge with width of $500 \mu \mathrm{m}$ and a trench. The melt residue of welding between the cap and the substrate can be clearly seen.

The three different bridges for the substrate were characterized and shown the bridge with a trench is the optimized geometry to balance heat and melt depth in laser welding. The bridge with a trench was implemented on a titanium wafer for large scale packaging. A $100 \mathrm{~mm}$ titanium wafer with thickness of $300 \mu \mathrm{m}$ was used as the substrate and cap in this work. Figure 9 presents the packaging process for both Si-MEMS and Ti-MEMS devices. The titanium cap and substrate were macro-machined as shown in Figure 9(a) and (b) respectively. Gold-ceramic-titanium or GCT feedthroughs were fabricated on the substrate and the MEMS devices were mounted and wire bonded to GCTs, Figure 9(c). Figure 9(d) shows the cap and substrate were brought together firmly by using a vacuum chuck. The laser parameters used to weld the cap to substrate shown in Table 1. Figure 11(d) shows a schematic of the packed device.

(a)

(b)

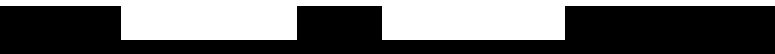

(c)

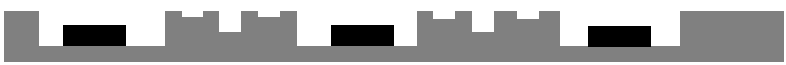

(d)

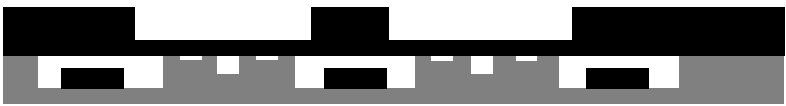

(e)

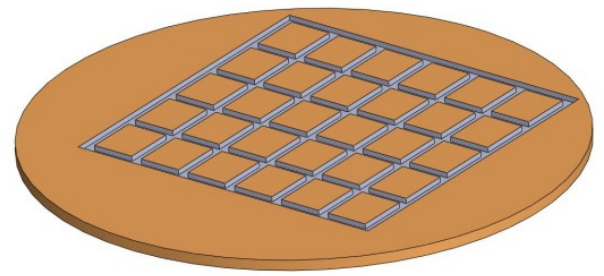

Figure 9: (a) and (b) Macro-machining a titanium wafer to fabricate the cap and substrate (c) Mounting the MEMS device on the substrate and wire bonding to GCTs (d) The cap and substrate are brought together by using a vacuum chuck (e) Schematic of the packaged wafer.

The hermiticity of the packaged device was measured by helium leaking rate test base on MIL-STD-883E standard. The helium leak detector was used in this work is Alcatel ASM 142 and the leaking rate of $3.45 \times 10^{-10} \mathrm{~atm} . \mathrm{cc} / \mathrm{s}$ for volume of $0.18 \mathrm{~cm}^{3}$ was measured.

Table 1: Laser parameters were used to weld the titanium cap to the substrate in wafer scale packaging.

\begin{tabular}{l|l|l|l}
\multicolumn{2}{c|}{ Laser Spot } & \multicolumn{2}{c}{ Seam Welding } \\
\hline Pulse shape & Rectangular & Welding speed & $0.85 \mathrm{~mm} / \mathrm{s}$ \\
\hline Peak power & $1.3 \mathrm{KW}$ & Focal spot size & $300 \mu \mathrm{m}$ \\
\hline Pulse duration & $1 \mathrm{~ms}$ & Pulse frequency & $14 \mathrm{~Hz}$ \\
\hline Energy per pulse & $1.3 \mathrm{~J}$ & Spot overlap & $80 \%$
\end{tabular}

To verify the proposed packaging method and its functionality on device scale, it was applied to package an AFM tips on titanium substrate. The device tested is a self-actuating self-sensing AFM cantilever tip (MPA-41100-S, Veeco Instruments) and is shown wire bonded to the GCTs, Figure 10 (a) and (c). By measuring the resonant frequency and quality factor before and after packaging as shown in Figure 10 (d) it is determined that the applied technique has no effect on the packaged device or GCT feedthroughs. 


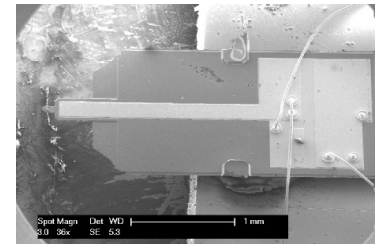

(a)

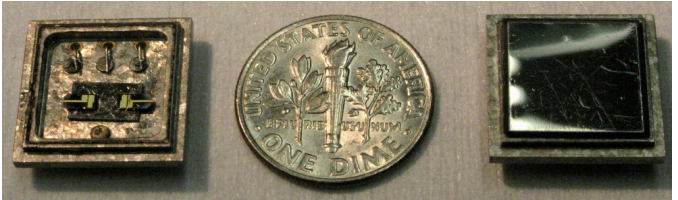

(c)

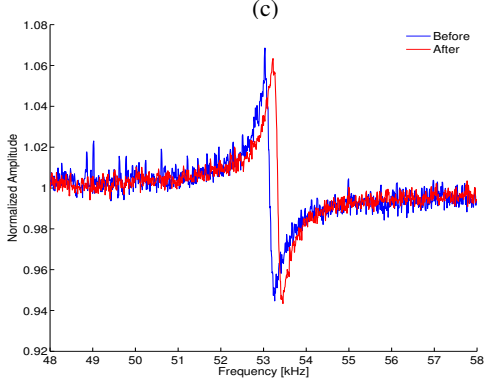

(d)

Figure 10: (a) AFM cantilever was used to measure resonance frequency and quality factor before and after packaging (b) GCT feedthtoughs $(c)$ the packaged device (d) resonance frequency of the AFM tips was consistence after and before packaging.

\section{CONCLUSION}

The new packaging method was modeled, simulated, fabricated and experimentally characterized. We combined titanium as a new material for packaging and YAG millisecond pulse laser as a localized heat source to introduce a new packaging technique for MEMS on both the wafer and device scale. The pulsed laser serves as a localized heat source and overcomes other packaging technique issues such a heat distortion and MEMS device damage from global heating packaging processes. The demonstrated packaging method applies directly to the titanium cap and substrate with no need of any interface material or films between them. This is considered a main advantage over the current methods. Our packaging method provides low cost, fast operation, high precision with low thermal distortion, reliable and biocompatible packaging for MEMS devices. This technique can be applied to both titanium and silicon MEMS making it a potential bridge between silicon Bio-MEMS and biotechnology applications.

\section{ACKNOWLEDGEMENT}

The authors would like to thank Veeco Instruments for providing the AFM tips.

\section{REFERENCES}

[1] H. Tachibana, K. Kawano, H. Ueda and H. Noge, "Vacuum wafer level packaged two-dimentional optic scanner by anodic bonding ", IEEE MEMS 2009, Sorrento, Italy.

[2] J.S. Mitchell and K. Najafi, "A detailed study and reliability for vacuum packages fabricated in a wafer-level Au-Si eutectic bonding process ", Transducer 2009, Denver, USA.

[3] M.F. Aimi, M.P. Rao, N.C. Macdonald, A. Zuruzi and D.P. Bothman, "High-aspect-ratio bulk micromachining of titanium", Nature Materials, 3, 103 (2004).

[4] D. Brunette, Titanium in Medicine, Springer, 2001.

[5] N.B. Dahotre, Laser Fabrication and Machining of Materials Springer Scinece, 2008, pp. 4-9.

[6] N. A.F.H Kaplan, "A model of deep penetration laser welding base on calculation of the keyhole profile", Journal of Physics D: Applied Physics 27:1805-1814

[7] V.N. Tokarev and A.F.H. Kaplan, "An analytical modeling of time dependent pulsed laser melting", Journal of applied physics, volume 86, number 5, September 1999.

[8] W.W. Duley, Laser Welding, Wiley, New York, 1999.

[9] Department of Defense, MIL-STD-883, "Test Method Standard, Micro-Circuts", methode 1014.9, March 14, 1995

\section{CONTACT}

*P. Bozorgi, tel: +1-323-206-1506; payam@engineering.ucsb.edu 